\title{
HTS SQUID Gradiometer Using Substrate Resonators Operating in an Unshielded Environment—A Portable MCG System
}

\author{
Yi Zhang, Norbert Wolters, Jürgen Schubert, Dieter Lomparski, Marko Banzet, Grigory Panaitov, \\ Hans-Joachim Krause, Michael Mück, and Alex I. Braginski
}

\begin{abstract}
We have demonstrated and verified the basic feasibility of performing magnetocardiographic (MCG) measurements without magnetic shielding when using a first-order electronic gradiometer with our novel dielectric substrate resonator rf SQUID's. The setup at the operation site involved adjustment of the gradiometer's baseline length and adaptive balancing. Our experimental portable system was tested in three environments differing in the level of electromagnetic interference.
\end{abstract}

Index Terms-Gradiometer, MCG, resonator, SQUID.

\section{INTRODUCTION}

$\mathbf{R}$ ECENTLY, we developed a first-order electronic gradiometer incorporating our novel dielectric substrate resonator rf SQUID's having high sensitivity [1]. In this work, we report on experimental verification that the system is well suited for performing MCG measurements in unshielded environment.

In the past ten years, a number of HTS SQUID gradiometers for recording of human magnetocardiography (MCG) in unshielded environment have been demonstrated. Reported were first and second order axial electronic rf SQUID gradiometers with mechanical balance adjustment [2], [3], and without [4], as well as dc SQUID gradiometers with planar first order gradiometric antennas [5]. However, in contrast to the presently tested concept, these instruments operated with a fixed baseline length and, in most cases, with conventional rather than adaptive balancing [6].

In this paper, we highlight the following new aspects of our work:

a) A new generation of rf SQUID magnetometers with dielectric substrate resonators suitable for operation in unshielded environment.

Manuscript received August 5, 2002

Y. Zhang, N. Wolters, J. Schubert, D. Lomparski, M. Banzet, G. Panaitov, and H.-J. Krause are with Institute of Thin Films and Interfaces (ISG-2), Research Center Jülich GmbH (FZJ), D-52425 Jülich, Germany (e-mail: y.zhang@fzjuelich.de).

M. Mück is with Institute of Applied Physics at the University of Giessen, D-35392 Giessen, Germany (e-mail: Michael.Mueck@ap.physik.unigiessen.de).

A. I. Braginski was with Institute of Thin Films and Interfaces (ISG-2), Research Center Jülich GmbH (FZJ), D-52425 Jülich, Germany (e-mail: abragins@nycap.rr.com).

Digital Object Identifier 10.1109/TASC.2003.813864 b) The optimization of the first order gradiometer baseline length for MCG measurements in conjunction with adaptive balancing.

c) The new readout electronics, integrated with microprocessor remote control by a serial RS 232 connection to a laptop computer.

d) Test MCG measurements at three sites in different countries: Germany, the United Kingdom and China, using our new portable instrument.

\section{SUBSTRATE RESONATOR CONCEPT}

The first order electronic gradiometer consists of two SQUID magnetometers. In order to achieve the required gradiometer sensitivity, one has to use magnetometers sufficiently sensitive over the signal frequencies of interest, down to below $1 \mathrm{~Hz}$ in the case of MCG. We have already shown in [1] that the instability of the operating point of an rf SQUID significantly increases the $1 / f$ noise. The rf SQUID operating point depends not only on the value of the rf current flowing in the tank circuit, but also on the coupling between SQUID and tank circuit, and thus on the mutual inductance $M$. The value of $M$ can be different in different environments. Nearby metal or dielectric objects can reflect or absorb rf energy and thus change the rf field distribution. Any motion or displacement of such objects changes then the value of the mutual inductance $M$ and thus affects the SQUID's operating point. As the environment is uncontrollable, confining the rf field distribution to the space close to the SQUID can substantially reduce the influence of the environment on $M$, improve the stability of operating point and thus reduce the $1 / f$ noise. Kornev et al. were first to show that a dielectric resonator having high permittivity $\varepsilon$ can be used to replace the conventional LC (coil and capacitor) tank circuit [7]. For confining the rf field to a small spatial volume, the $\mathrm{SrTO}_{3}$ is a suitable material since its permittivity $\varepsilon$ is approximately 2000 at $77 \mathrm{~K}$.

We have developed a very practical concept - the single substrate dielectric resonator [1]. A standard $\mathrm{SrTO}_{3}$ substrate of 10 $\mathrm{mm} \times 10 \mathrm{~mm} \times 1 \mathrm{~mm}$ is a dielectric resonator replacing the conventional tank circuit. A thin YBCO film is deposited onto it and the flux focuser patterned. A small rf washer SQUID with a step-edge junction is pressed against the focuser in flip-chip geometry to attain suitable coupling. The resonance frequency $f_{0}$ of the dielectric resonator with the YBCO film flux focuser structure is about $650 \mathrm{MHz}$. 


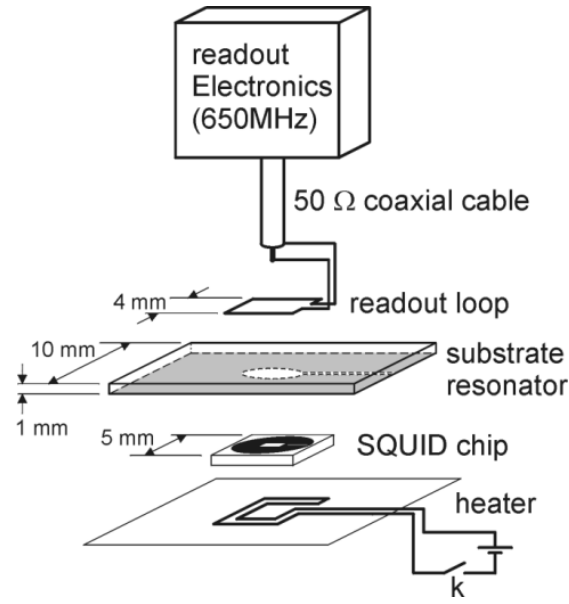

Fig. 1. Schematic drawing of the dielectric substrate resonator and SQUID in flip-chip configuration, the readout loop and heater, which are all contained in sealed fiberglass capsule.

In the flip-chip configuration, the planar thin film SQUID is placed between two substrates with high $\varepsilon$, and the rf energy is confined to within these substrates. Because only little rf energy is leaking out of the resonator-SQUID stack, the mutual inductance $M$ is much less dependent on the environment than, for example, in the coplanar resonator configuration [8]. Hence, the $1 / f$ noise is substantially reduced. This is a dielectric analogy to the, so-called, toroidal bulk rf niobium SQUID structure demonstrated in the 1970s [9], [10].

Fig. 1 shows schematically the flip-chip configuration used and the arrangement of components inside a sealed fiberglass capsule. A readout (coupling) loop etched in a printed circuit board is positioned on the uncoated side of the resonator substrate to couple the SQUID to the readout electronics via a $50 \Omega$ coaxial cable. A planar heater is placed on the SQUID chip as well. The encapsulation not only protects the YBCO thin film from moisture, but also reduces the low frequency noise due to temperature fluctuations caused by bubbling nitrogen. Such SQUID magnetometers achieve a very low noise level. We measured white noise levels of about $24 \mathrm{fT} / \sqrt{ } \mathrm{Hz}$. At 10 and $1 \mathrm{~Hz}$, the noise is lower than 40 and $100 \mathrm{fT} / \sqrt{ } \mathrm{Hz}$, respectively.

\section{Choosing the Optimum Baseline Length}

The goal of practical MCG measurements is to attain the highest possible signal-to-noise ratio (SNR). Using sensitive SQUID magnetometers, one must also minimize the magnetic noise (external spurious signals) from the environment. In our approach, we adaptively adjust the balance, select the filters and optimize protection against the high-frequency electromagnetic interference. In this paper, we focus on the optimization of the gradiometer baseline length for a given signal source type, i.e., the heart signal of a given human subject.

Fig. 2 shows our SQUID holder. The two encapsulated SQUID magnetometers are mounted in an axial configuration. The bottom SQUID (close to the measured signal source) is fixed, whereas the position of the top SQUID can be changed. The baseline length, $b$, is adjustable from 2 to $20 \mathrm{~cm}$. The optimum $b$ can be selected from a series of measurements with varying $b$, when setting up at a given location. In the present

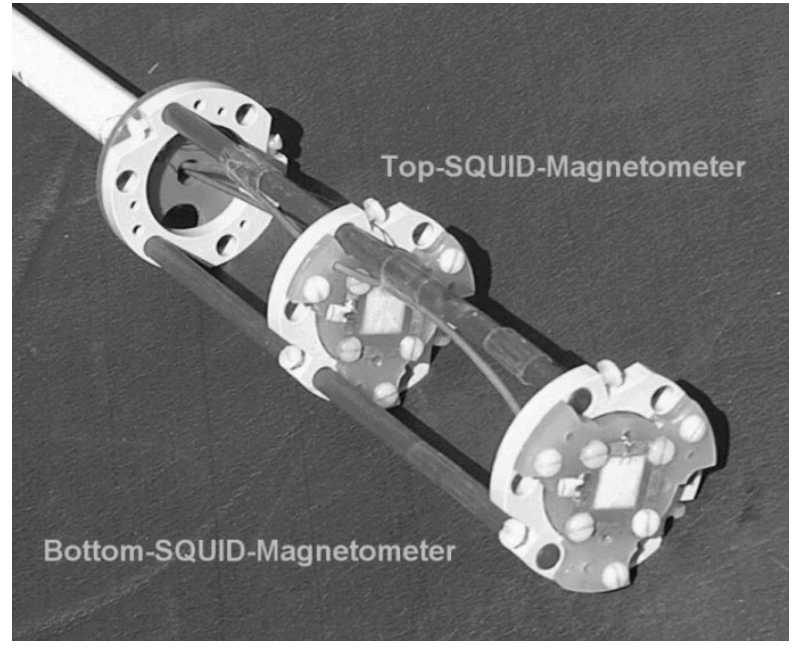

Fig. 2. A photograph of the SQUID holder. The bottom SQUID is fixed, the position of the top SQUID can be adjusted.

demonstrator instrument, $b$ is adjusted after warming up to the room temperature, while a practical clinical system could be designed to allow adjustment from outside the cold cryostat.

We first determined the effect of baseline length on the amplitude of the measured output signal of the gradiometer, as compared to the signal of the bottom magnetometer. We modeled the magnetic activity of human heart by currents in a coil of 8.3 $\mathrm{cm}$ diameter, generating magnetic field signals at a distance of $14 \mathrm{~cm}$ to the bottom SQUID. At this distance, the peak signal (R-wave) of a typical human heart produces a field at the bottom SQUID of about 50 pT. For baseline lengths of about 3, 5, 10 and $15 \mathrm{~cm}$, the respective output signal of our gradiometer is about 50, 60, 80 and $90 \%$ of the magnetometer signal, respectively. Indeed, it is known that, in order to obtain a large MCG signal, a gradiometer baseline length should not be too short.

Subsequently, to find out how to eliminate the noise from the environment, we have performed measurements with our MCG simulator coil in the unshielded environment of our laboratory, with different $b$ settings of our gradiometer and adaptive balancing to maximize SNR. The time series filtered by a low-pass filter to a bandwidth of $30 \mathrm{~Hz}$ are shown in Fig. 3(a). In the plot of Fig. 3(b), we show that the SNR vs. baseline length is not the expected curve with only one maximum. The dominant noise was not the intrinsic noise of our system, but rather the $162 / 3 \mathrm{~Hz}$ interference from the German railway. This disturbance cannot easily be filtered because it lies within the major MCG signal frequency range. The use of a notch filter would either cause signal distortions or result in signal loss [4]. This spurious signal may have a large spatial phase change. Therefore, we observed more than one maximum in the SNR as the baseline length was varied. In our environment we obtained the best SNR with a baseline length of $14 \mathrm{~cm}$. In contrast, the lowest SNR was observed for baseline lengths of 5 to $6 \mathrm{~cm}$, which until now were typically used in HTS MCG gradiometers [2]-[5].

In most cases, the overall noise of the first order gradiometer system is not given by the theoretical level, $\sqrt{ } 2 B_{N}$, where $B_{N}$ is the magnetic field resolution of one magnetometer. In our work, the noise was often found to be determined by strong interference from either the railway or nearby electric power 

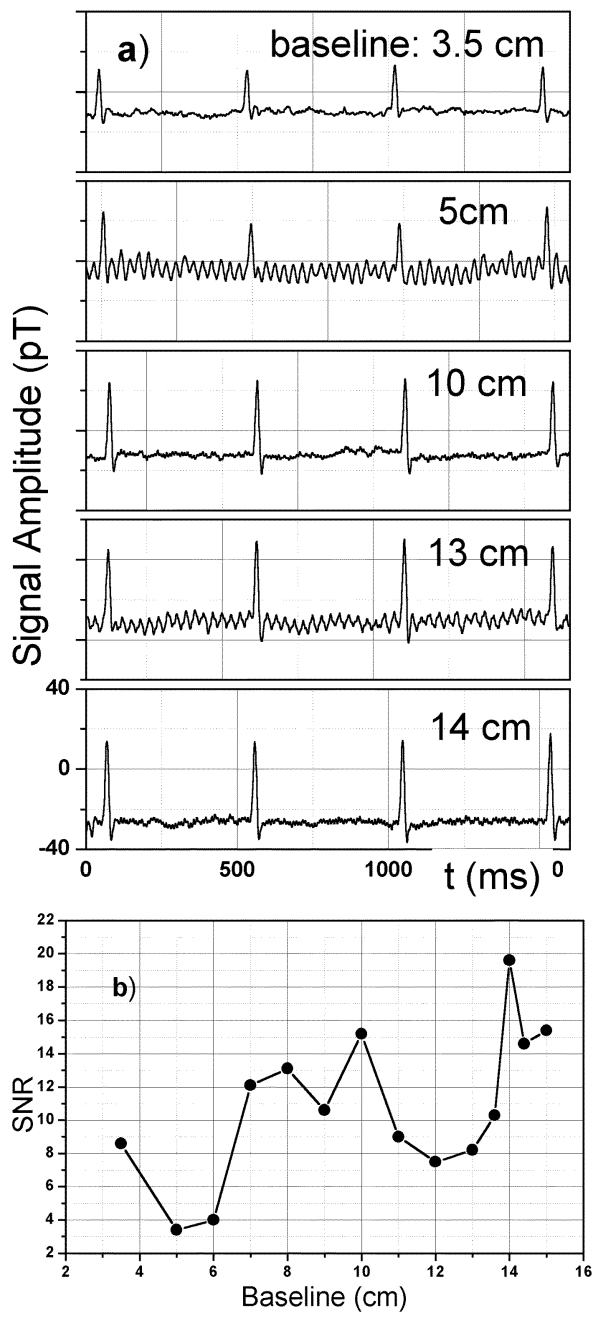

Fig. 3. (a) Real time trace (source: coil driven by current source simulating an MCG), recorded in unshielded environment with different baseline lengths. The video bandwidth was about $30 \mathrm{~Hz}$. (b) Signal-to-noise ratio vs. baseline length. With a baseline length of $14 \mathrm{~cm}$, we obtained the best SNR for MCG measurements in our environment.

plants. Indeed, a simple noise measurement of the SQUID gradiometer in a quiet environment has no practical relevance. The optimum baseline length for the best SNR depends on the measured subject and also on the environment.

\section{REAdOUt ELECTRONICS}

Fig. 4 shows our portable SQUID gradiometer demonstrator system for MCG feasibility testing. The readout electronics is mounted on top of the liquid nitrogen cryostat.

The tripod holding the cryostat can be dismantled for transportation. The complete system fits into a traveling suitcase (measuring $0.75 \mathrm{~m} \times 0.5 \mathrm{~m} \times 0.28 \mathrm{~m}$ ).

The readout electronics is designed for two SQUID magnetometer channels and also contains the summation unit and filters. It is integrated with a microprocessor remote control via a serial RS 232 connection to a laptop computer. Even nonexperts can operate the system. The user program interface, shown in Fig. 5, permits the operator to automatically or manually adjust

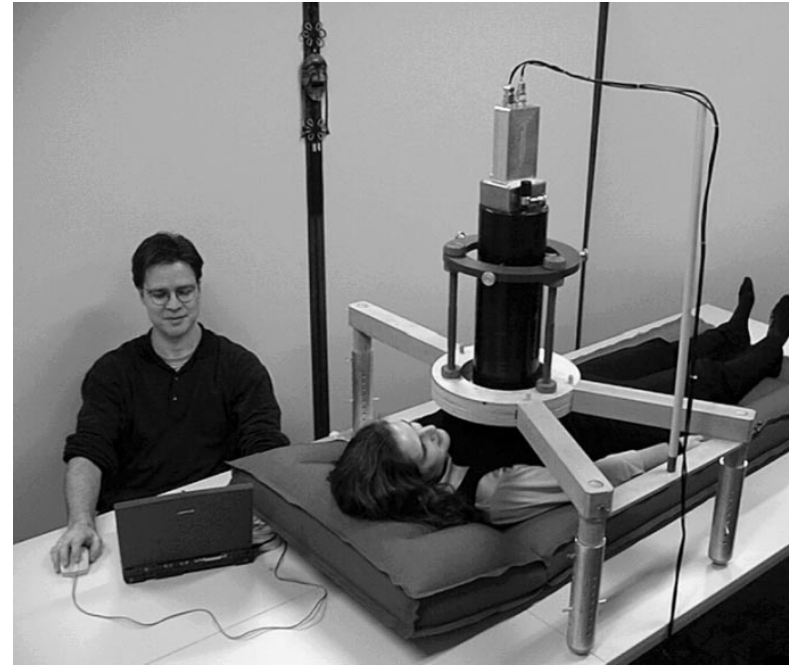

Fig. 4. A portable SQUID gradiometer system for MCG measurements (our demonstrator). The tripod holding the cryostat may be dismantled for easy transportation. The complete system fits into a travel suitcase.

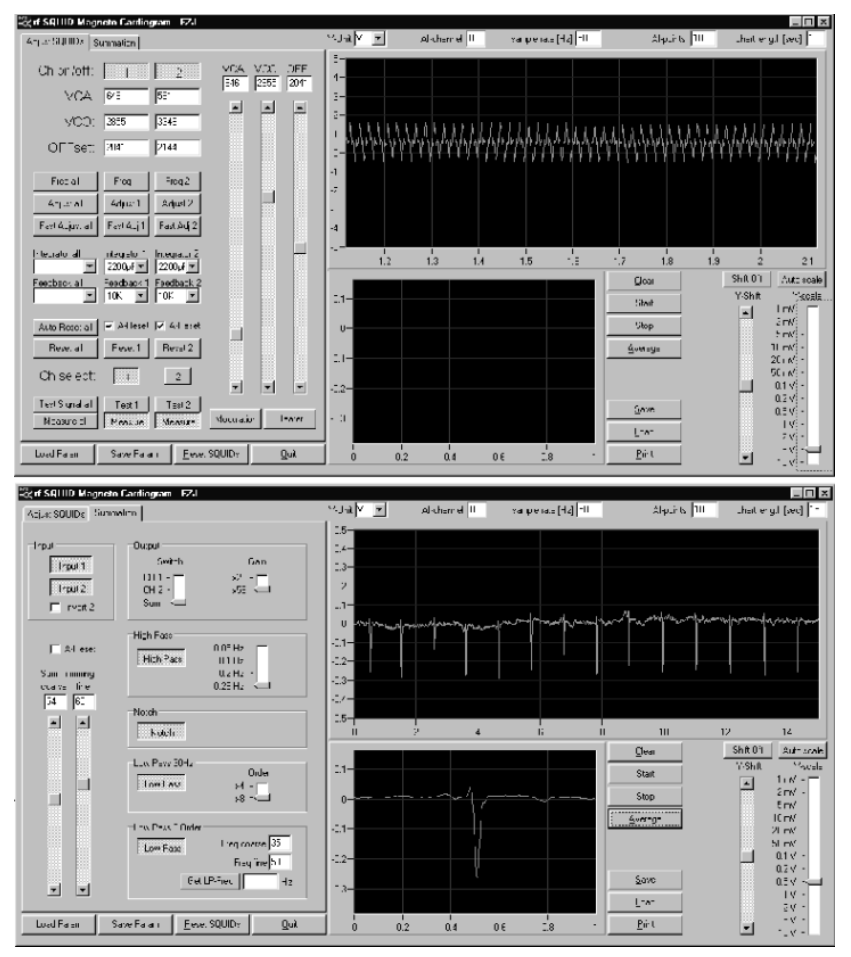

Fig. 5. The user program interfaces allow manual and automatic adjustment of the SQUID's working points (upper part), the balance (summation), as well as the selection of the filters. The measured real-time MCG time series is displayed on the top display. The averaged cardiac cycle is shown on the lower display.

the SQUID's operating points (upper part of Fig. 5), the adaptive balance (summation), as well as the selection of the filters. The measured real-time MCG time series is shown on the top display. The averaged cardiac cycle is presented on the lower display. The measured MCG signals can be saved and printed out on paper.

\section{MCG Test MEASuREMENTS}

Using the instrument described above, we performed MCG measurements at different sites in different countries. Examples 


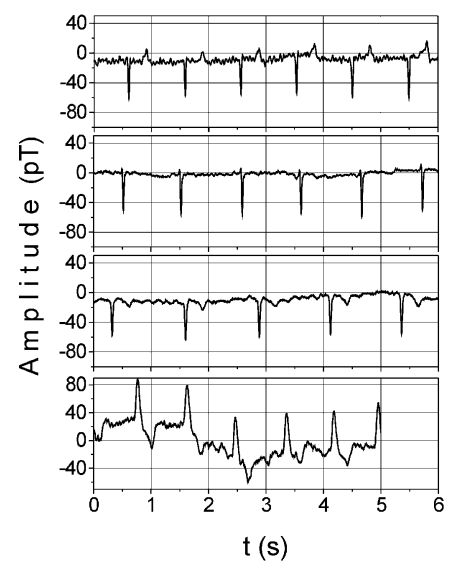

Fig. 6. MCG measurements at different sites in Germany, England, Scotland, and China. The video bandwidth was about $30 \mathrm{~Hz}$.

of recorded data are shown in Fig. 6. The top time series was recorded in our laboratory. The one immediately below it was recorded at NPL in London, England, and the third below at a hospital in Glasgow, Scotland. The bottom one, most recent, was recorded in the exhibition hall of the International Trade Center in Beijing, during a trade fair. The first three measurements were made for healthy human subjects, the fourth is for a subject with a known heart disease, a so-called atrial septal defect. The environment in the exhibition hall in Beijing was extremely noisy. The $50 \mathrm{~Hz}$ signal was about $0.8 \mu \mathrm{T}$ (peak to peak), about 20 times larger than in a typical laboratory environment. During the one-week exhibition, we did not have to perform any readjustment of our system. These measurements demonstrate the feasibility of performing MCG recordings even in very noisy environments.

\section{CONCLUSION AND OUTLOOK}

We have demonstrated that a first-order portable HTS gradiometer utilizing substrate resonator rf SQUID's can be a suitable base for future development of commercial multichannel MCG systems. Such future systems should incorporate (i) an externally adjustable baseline length optimized during the instrument's setup at the site of operation, and (ii) adaptive, auto- mated gradiometer balancing. We have also demonstrated that HTS rf SQUID magnetometers using dielectric substrate resonators may be used in an unshielded and very noisy environments.

While the technology of LTS SQUID systems for MCG is relatively mature, that of HTS systems is not. The fabrication technology of thin-film YBCO Josephson junctions is not sufficiently well developed, and YBCO wire suitable for gradiometers is not available. With electronic gradiometers it is difficult to construct systems having a very large number of measurement channels. However, we believe that fully automated HTS rf SQUID gradiometer systems with up to 9 measurement channels could be usable in the near future. It would be advantageous to have such system miniaturized to demonstrate the additional advantages of HTS SQUID systems - portability and the operating cost.

\section{ACKNOWLEDGMENT}

The authors thank L. Hao, J. Macfarlane, J. Gallop, C. Carr, G.B. Donaldson and the Peking University for the cooperation and the help in performing the MCG measurements.

\section{REFERENCES}

[1] Y. Zhang, J. Schubert, N. Wolters, M. Banzet, W. Zander, and H.-J. Krause, "Substrate resonator for HTS rf SQUID operation," Physica C, 2002, to be published.

[2] Y. Tavrin, Y. Zhang, M. Mück, A. I. Braginski, and C. Heiden, Appl. Phys. Lett., vol. 62, pp. 1824-6, 1993.

[3] J. Borgmann, P. David, G. Ockenfuss, R. Otto, J. Schubert, W. Zander, and A. I. Braginski, Rev. Sci. Instrum., vol. 68, pp. 2730-4, 1997.

[4] Y. Zhang, G. Panaitov, S. G. Wang, N. Wolters, R. Otto, J. Schubert, W. Zander, H.-J. Krause, H. Soltner, H. Bousack, and A. I. Braginski, Appl. Phys. Lett., vol. 76, pp. 906-8, 2000.

[5] K. A. Kouznetsov, J. Borgmann, and J. Clarke, Appl. Phys. Lett., vol. 75, pp. 1979-81, 1999.

[6] J. Vrba, "SQUID gradiometers in real environments," in SQUID Sensors, H. Weinstock, Ed. Dordrecht: Kluwer, 1996, pp. 63-116.

[7] V. K. Kornev, K. K. Likharev, O. V. Snigirev, Y. S. Soldatov, and V. V. Khanin, Radio Eng. Electronic Phys., vol. 25, pp. 122-4, 1980.

[8] Y. Zhang, W. Zander, J. Schubert, F. Rüders, H. Soltner, M. Banzet, N. Wolters, X. H. Zeng, and A. I. Braginski, Appl. Phys. Lett., vol. 71, pp. 704-6, 1997.

[9] W. L. Goodman, V. W. Hesterman, L. H. Rorden, and W. E. Goree, Proc. IEEE, vol. 61, p. 20, 1973.

[10] R. Rifkin and B. S. Deaver, Phys. Rev., vol. B13, p. 3894, 1976. 